REVISTA NOMADÍAS

Noviembre 2011, Número 14, 79 - 97

\title{
De La Pedagogía Cívico-Sexual al Gobierno de los Cuerpos
}

\section{From Civic and sexual Pedagogy to the Government of the Bodies}

\author{
Mary Luz Estupiñán Serrano \\ Universidad de Chile \\ maryluzestupinan1@gmail.com
}

\begin{abstract}
RESUMEN
El presente texto pretende develar los discursos subyacentes sobre sexualidades y ciudadanías presentes en los Planes y Programas de Educación Sexual y Construcción de Ciudadanía en Colombia, surgidos a partir de los años noventa. Para ello revisamos las producciones discursivas con respecto a estas problemáticas con el fin de determinar los aspectos de estas cadenas significantes - premisas, enunciados, conceptos y vocabulario-que se ponen al servicio de la disciplina y el control de los cuerpos y los comportamientos. Más allá de las bondades que estos proyectos encarnan, este texto focaliza los discursos que vienen aparejados y que afectan directamente los procesos de construcción de subjetividades juveniles.
\end{abstract}

\begin{abstract}
This article tries to reveal the underlying discourses on sexualities and citizenships at the back of the Plans and Programs of Sexual Education and Construction of Citizenship in Colombia since Nineties. To do this we reviewed the discursive productions with regard to these issues in order to determine the aspects of these chains of meaning - premises, statements, concepts and vocabulary- that are in favor of discipline and control of the bodies and behaviors. Beyond the advantages that these programs support, this article focuses the discourses that come behind and that directly affect the processes of construction of youthful subjectivities.
\end{abstract}

Palabras claves: discursos, sexualidades, ciudadanías, educación, Colombia. 
Keywords: discourses, sexualities, citizenships, education, Colombia.

Desde la confiscación de la sexualidad al cuarto privado de la familia conyugal, ha sido difícil devolverle la espontaneidad a los cuerpos, evitar los modelos, desaconsejar la mesura, así como liberar los discursos de la economía libidinal. La pareja establecida por un varón y una mujer continúan en el trono relacional y la procreación sigue siendo el deseo por excelencia -aunque no de la misma forma e intensidad como lo pretendió la sociedad burguesa estudiada por Foucault-. Pese a que "nuevos" relacionamientos y "nuevas" sexualidades han obligado a los discursos y las prácticas a flexibilizarse, dichas normas continúan en la trastienda discursiva dominante, puesto que siguen al servicio de la política, la economía y la religión.

En este sentido, el Estado -en tanto institución- se vale de sus aparatos políticos ${ }^{2}$ para afectar la vida privada de los sujetos con sus fuerzas normativas. Es a través de la ciudadanía que se modela dicha economía libidinal pues a partir de los procesos de concientización de las prácticas modeladas -procesos posibles gracias a ciertos dispositivos pedagógicos y educativos- se pretende establecer los comportamientos sexuales deseables para todas y todos. Sin embargo, la construcción histórica y cultural de las sexualidades y los cuerpos, han posibilitado, en parte, el desmantelamiento de los discursos y las prácticas discursivas naturalistas y esencialistas sobre los mismos, otorgándoles un carácter dinámico, pero ¿hasta dónde el sistema educativo colombiano contribuye, o puede contribuir, a ello?

Para nuestro análisis definiremos "sexualidades", así, en plural, para evidenciar que no hay una única forma de referirla, expresarla, practicarla y sentirla. De esta manera, las sexualidades serán entendidas como: "construcción[es] histórica[s], que reúne[n] una multitud de distintas posibilidades biológicas y mentales -identidad genérica, diferencias corporales, capacidad reproductivas, necesidades, deseos y fantasías- que no necesariamente deben estar vinculadas, y que en otras culturas no lo han estado" (Weeks, 1986, 20).

Respecto a la ciudadanía, ésta también será pluralizada puesto que es "una categoría densa, porque es histórica, porque es compleja y, sobre todo, porque no puede reducirse ni a un concepto, ni a un personaje, ni a una fantasía, ni a un derecho, ni 
a una pertenencia, ni a una existencia (Cullen, 2007, 42). La tarea a emprender en estos dos campos, que sin duda se entreveran, es la comprensión (Weeks, 1986; Foucault, 2003). La vía que nos permitirá acercarnos a esta tarea será el análisis de discurso en términos foucaultianos: "los discursos son prácticas que responden a ciertas reglas de formación [históricas, políticas, sociales y culturales] que los constituyen y posibilitan" (Colombani, 2009, 29). Por tanto, el asunto está en atender a "las irrupciones; la irrupción del conjunto de enunciados, prácticas y discursos que una determinada época valida como verdaderos porque se aglutinan y organizan conforme a una cierta coherencia" (Colombani, 2009, 30). Lo que está aquí como trasfondo es una relación entre discurso y poder que, todavía, estaría sustentada por unas instituciones y sus correlatos tecnologizantes: leyes, normas, enunciados científicos, filosóficos y mediáticos, pautas culturales, sociales y morales. De ahí nuestro interés en los planes, programas e iniciativas de educación estatales, pero también en las propuestas de otros entes al margen de la estatalidad, para revisar cómo éstos responden a las omisiones y al discurso dominante.

En este texto me remitiré a parte de los hallazgos de la investigación discursiva que refiere a premisas, conceptos, enunciados y vocabulario que hacen parte de la cadena de significación sobre las sexualidades y las ciudadanías contenidos en planes, programas e iniciativas educativas en Colombia a partir del año noventa, puesto que es la década en la que se inicia la discusión sobre su incorporación conceptual al currículo educativo.

Si bien esta pesquisa fue concluida en septiembre de 20093, esperamos contribuir con nuestros alcances a la discusión en torno a los programas de educación sexual que actualmente se discuten en Chile, no sólo en cuanto a los discursos manifiestos o latentes sino también respecto a las perspectivas que quedan fuera, es decir, aquello que no entra en la economía libidinal de las políticas educativas. Aquí cabe resaltar que opciones como la no maternidad o no paternidad siguen sin siquiera mencionarse en las iniciativas analizadas.

\section{Discursos sobre sexualidades}

A través de la revisión del material que reúne documentos tanto oficiales ${ }^{4}$ como las denominadas propuestas "alternati- 
vas $^{\prime \prime}{ }^{5}$, fue posible establecer que los discursos que han predominado con respecto a las sexualidades, especialmente, en los planes estatales de Educación Sexual de los años noventa en Colombia, son los científicos o biologicistas y los psicologicistas.

Estas perspectivas, aunque se muestren como técnicas o "neutras axiológicamente" (Cáseres, 2004), proponen una aproximación determinada, esto es individualizante y normativa en términos de sexualidad juvenil y adolescente -en pocos casos y experiencias se hace mención a la sexualidad infantil-. Con ello se pretende generar en cada "sujeta/o" capacidades / competencias para evaluar sus comportamientos sexuales con lógicas prescriptivas. Aunque estas competencias vayan encaminadas supuestamente al ejercicio de una sexualidad "libre y satisfactoria", frecuentemente van aparejadas de dosis de control (y no tanto de disciplinamiento), así la sexualidad también implica un ejercicio "responsable y sano" (Viveros, 2006), generando de esta manera espejismos de libertad (o libertad tutelada), adherida siempre a restricciones que requieren -ahora- del consentimiento de las/los sujetos para una relegitimación.

Esta trampa corresponde, en términos foucaultianos, a la capacidad de estructurar el campo de acción del/los otro/s. Aquí el centro cambia de lugar (de afuera hacia adentro) así como de sujetos (de adultos a jóvenes) y el poder como dominación se traslada a una autosoberanía en la que ella/él misma / o interviene en la potestad de sus acciones posibles. En otras palabras, es la normalización y modulación de las prácticas y de los estilos de vida con el fin de dominarlos, limitar las libertades e inmovilizar la potencia creadora.

En este sentido, dentro del discurso biologicista, encontramos las perspectivas médicas y ginecológicas que se instalaron en los programas de sexualidad desde los años $70^{6}$. No obstante, hay que reconocer que el tratamiento que tuvo la sexualidad en los años 70 y 80, amplía su horizonte de expectativas en la década siguiente, incorporando los avances científicos, por un lado y aprovechando las laxitudes sociales construidas con la incorporación de nuevos métodos de planificación familiar, por otro. Empero, la biología y la medicina continúan dominando el campo de las sexualidades, pues su saber-poder no ha sido mermado, ni develado, por lo menos no de manera explícita, lo que implica que la mirada clínica continúa ejerciendo su hegemonía sobre la sexualidad. 
El predominio de estos discursos se constata en el acercamiento que se hace con respecto al sexo, el cuerpo y los derechos sexuales y reproductivos, en los que priman los aspectos fisiológico-anatómicos, el cuidado (no de sí desde sí, como deseó Michel Foucault, sino desde una/s norma/s exterior/res), la prevención y la salud sexual. En este sentido, se enfatiza principalmente la información sobre los riesgos del ejercicio sexual, focalizando los embarazos adolescentes y las infecciones y enfermedades de transmisión sexual (ITS-ETS) y, por supuesto, la pandemia del VIH/SIDA (MEN, 1993, 1999). De ahí la importancia de pensar estos discursos, pues continúan privilegiando y/o poniendo en desventaja a sujetas/sujetos y funciones específicas, a saber: las mujeres y su capacidad reproductiva y la población LBGTI $^{7}$ al igual que los eventuales riesgos de VIH-SIDA.

Una breve revisión a la continuidad de estos discursos, permitirá ilustrar lo señalado. En el año 1992, en la antesala de la formulación del Primer Plan Nacional de Educación Sexual (PNES, 1993), liderado por el Programa Presidencial para la Juventud, la Mujer y la Familia (PPJMF), se reconoce la necesidad de "institucionalizar la educación sexual mediante programas formales integrados al currículo de nivel post y pre-universitario que implementen la capacitación y especialización en el área sexológica tanto en investigación como en terapia y educación sexual" (PPJMF, 1992, 42). En 1999, en la actualización del plan anterior por parte del Ministerio de Educación Nacional (MEN), se mantienen los ejes propuestos para articular las temáticas que se deben desarrollar a la largo de todos los grados. Estos ejes son: persona, pareja (HOMBRE-MUJER) ${ }^{8}$, familia y sociedad. Para ejemplificar, dentro del eje de persona para el grado $5^{\circ}$ del nivel de primaria se pretende "prepararlos [estudiantes varones y mujeres] para la pubertad"; luego en el grado $7^{\circ}$ de educación secundaria, se propone abordar "el cuidado del cuerpo", "los cambios físicos de la pubertad", "la menstruación y la eyaculación" y para el grado $9^{\circ}$ se promueve la responsabilidad para "la prevención de Enfermedades de Transmisión Sexual y VIH/ SIDA y de embarazos no deseados" (MEN, 1999).

En el año 2006, se planea un Proyecto Piloto de Educación Sexual y Construcción de Ciudadanía (PESCC Convenio MENUNFPA), el cual cambia la lógica de los ejes por los hilos conductores. El hilo conductor en Derechos Sexuales y Reproductivos propone, a manera de ejemplo, que las y los docentes" "promue- 
van el conocimiento, la comprensión y el empoderamiento necesarios para que toda persona sepa acceder a servicios de Salud Sexual y Reproductiva de calidad (incluyendo anticoncepción, atención a disfunciones sexuales, ITS, VIH/SIDA, etc.)" (MEN, 2006, 57).

Para ello las / los estudiantes deben desarrollar competencias que deben ser expresadas de la siguiente manera: “Comprendo el funcionamiento biológico de la sexualidad y de la reproducción humana, y esta comprensión me sirve para vivir una sexualidad satisfactoria y saludable para mí y para los demás" (MEN, 2008, $15)^{10}$. Es importante destacar aquí el énfasis en la "reproducción humana", pues ello acentúa una idea de la sexualidad normativa y dominante (biologicista), donde el placer del sexo por el sexo es descartado en pos de la perpetuación de la especie.

Así, la sexología y la medicina son las voces autorizadas para un acercamiento al cuerpo y a los comportamientos que de esta relación emergen. Por tanto, son las encargadas de realizar terapias y dar consejos que regulen los patrones de comportamiento social e individual, pues los cambios corporales y psíquicos poco comprendidos en el mundo de la vida y envueltos en un halo de enfermedad deben ser llevaderos. Para ello la escuela complementa este andamiaje discursivo que debe hacer posible la prevención de enfermedades, embarazos prematuros y abortos así como control de las parafilias, las "disfunciones sexuales" y las enfermedades de transmisión sexual. En esta vía, la educación sexual permite comprender la reproducción humana y el funcionamiento biológico de los cuerpos para desembocar en prácticas religiosamente correctas que permitan llegar a los puertos indicados de la pareja, la familia y así contribuir a la consolidación de una sociedad "armónica".

Estar informados es, sin duda, un requisito básico que potencia la acción. No obstante, apropiarse del cuerpo es principalmente deshilvanar la madeja de discursos e instituciones que lo cruzan, lo controlan y que limitan sus potencialidades creativas e innovadoras de las que hablaba Foucault, sobre todo en $\mathrm{La}$ inquietud de sí. No es posible desligar el cuerpo de la heterosexualidad obligatoria, la maternidad y la familia nuclear, discursos con dispositivos propios, institucionalizados al servicio de un orden político del que son ya bien reconocidos los efectos producidos sobre determinados sujetos a través del tiempo (Rich, 1999 [1981]). 
El control de la natalidad, la prevención de los embarazos no deseados y las enfermedades e infecciones de transmisión sexual y la promoción de la planificación familiar se han convertido en los mecanismos privilegiados para normalizar la reproducción de las poblaciones puesto que "el gobierno de la sexualidad de los jóvenes [adolescentes y sujetos excluidos del orden hegemónico] requiere tanto de las disciplinas individuales del cuerpo y su acción correccional como de las regulaciones colectivas de su sexualidad" (Viveros, 2006, 153).

De otro lado, los discursos desde los grupos sexuales emergentes se focalizan en la categoría de sexo. Sus discursos toman gran importancia puesto que las representaciones sociales y culturales que derivan de la aceptación de sus dos bases corpóreas perjudican profundamente sus posibilidades de acción, debido a que las asignaciones tradicionales subrayan exclusivamente el carácter material y funcional del sexo, esto es, lo anátomo-fisiológico, que diferencia a varones de mujeres, sin dar paso a las combinaciones, los cruces y las nuevas figuraciones que podrían resultar cuando no se atiene a lo normativo (Foucault, 2005; Lamas, 1996; Butler, 2000):

El sexo es una distinción entre hembras y machos basada en las grandes regularidades de correspondencia en los cuerpos humanos entre tres componentes del mismo: el sexo cromosómico o genético (alelos XX o XY), el sexo hormonal (carga diferenciada de hormonas femeninas y masculinas en todas las personas) y el sexo anatómico (pene o vulva al momento del nacimiento, y desarrollo de los caracteres sexuales secundarios a partir de la pubertad)... pero la propia naturaleza exhibe casos de menor correspondencia entre los componentes referidos que hacen difícil la asignación social del sexo, es decir, en casos de intersexualidad (García, 2007, 16-17).

Elementos como cromosomas, hormonas, y aparatos anatomo-fisiológicos hacen difícil sacar la categoría sexo del dominio científico. Esto además ha generado representaciones y respuestas dogmáticas contra las cuales las resistencias también han logrado ciertas resignificaciones. Ello nos lleva a señalar que el sexo-cuerpo (naturaleza) es cultural, aunque no "meramente cultural" como subraya Butler (2000) y de su develamiento dependen muchos debates por venir en pos de una sexualidad realmente libre.

Aunque no significa negar estos discursos, las variaciones, las traslapaciones y las ambigüedades sexuales, deben dar paso 
a la creación de estilos de vida distintos, incluso dar paso a una cultura distinta (Foucault, 2005). Pues para los sujetos intersexuales, los discursos biologicistas constriñen aún más sus posibilidades de visibilización y reconocimiento ya que su ambigüedad genital, es decir, la presencia simultánea de los dos órganos genitales (pene y vulva) con un cierto grado de desarrollo dentro de un mismo cuerpo -denominado "hermafroditismo verdadero" en el discurso médico- constituye una "anormalidad" desde el punto de vista científico y social, y la solución viable en cualquiera de los dos casos es el quirófano para responder a los mandatos sociales de "heterosexualidad obligatoria", pasando por encima de las voluntades individuales (García, 2007; Rich, 1999)

En el mismo sentido, el discurso científico aún privilegia el tema de la epidemiología y la salud pública, y desde este punto de vista, la aproximación a lo sexual es positivista como lo afirma Carlos Cáseres (2004), aunque fluctúa entre una ambivalencia moral y pragmática puesto que puede asumir políticas que privilegian la "reducción de daños" o riesgos frente a la inhibición/autogobierno "por principio", palpable en el tratamiento de la prevención de SIDA, las ITS/ETS: “Es posible, entonces, que el mayor potencial libertario de las prácticas hegemónicas de epidemiología y salud pública esté en esa tradición de pragmatismo benefactor. Porque al abrirse a lo socialmente excluido, y al definir fórmulas para su abordaje, la epidemiología contribuye a su legitimación" (Cáseres, 2004, 43).

No resulta tan paradójico que la respuesta estatal a las epidemias, las enfermedades y las "desviaciones sexuales" haya permitido "cierta" visibilización e incorporación de muchas categorías social y sexualmente excluidas en las políticas y programas estatales. Antonio Gramsci ya lo señaló con bastante antelación, pues los poderes hegemónicos han diseñado estrategias para desmontar las resistencias/contrahegemonías y la vía ha sido incorporarlas, darles un lugar dentro de su discurso. En efecto, esta lógica también ha jugado a favor de las presiones, demandas y luchas de los grupos "emergentes", cuya legitimación se empieza a dar en el marco de derechos que anuncian todas las propuestas revisadas en esta investigación. Ampararse en estas máscaras no es lo que ellas y ellos desearían, pero es el punto de fuga que permiten los discursos jurídico-legales, barrera que hay que franquear para alcanzar una participación política e intervenir en las decisiones que las/los afecta directamente. 
En suma, es evidente que el discurso biologicista es relevante en la medida que ofrece herramientas científicas para comprender las respuestas que varones y mujeres presentan frente a ciertas situaciones. No obstante, no hay que sobredimensionarlo puesto que dichas respuestas también se ven afectadas por una serie de factores sociales, históricos y culturales que además contribuyen a la producción y configuración de dichas respuestas, las cuales pueden no obstante ser descodificadas y transformadas.

Por otra parte, la intervención social con respecto a la salud sexual y reproductiva de los jóvenes en Colombia expresa lo que Didier Fassin y Dominique Memmi (2004) siguiendo a Foucault, llaman el "gobierno de los cuerpos", es decir, "la intromisión de los poderes públicos en la relación privada del individuo con su destino físico a través de códigos, reglamentos, normas, valores, relaciones de autoridad y de legitimidad" (Cit. en Viveros, 2006, 149).

De esta manera, los discursos psicologicistas se presentan en dos líneas: 1) cambio conceptual para buscar remover comportamientos y actitudes y 2) mecanismos o estrategias recomendadas o utilizadas para lograrlo (Cáseres, 2004). En la lógica de las políticas de prevención el Programa de Profamilia Joven ${ }^{11}$, tiene un horario de atención en consultorio pero también ofrece tallares educativos en los colegios a través de los cuales se entregan condones y se realizan muestras gratis de métodos de planificación, se ofrecen sesiones de consejería y pruebas voluntarias tanto de embarazo como de VIH/SIDA (y todo el paquete de servicios a bajo costo), mecanismos con los cuales pretenden cambios comportamentales sostenibles en el tiempo puesto que la repetición de estas lógicas empiezan a penetrar los cuerpos de las y los sujetos con su aparente aprobación, pero están diseñados, justamente, para producir tales efectos.

En este mismo orden de ideas, los discursos encaminados a la construcción del cuerpo en la escuela configuran mecanismos de auto-vigilancia, hetero-vigilancia y co-vigilancia, en donde se involucra el sí mismo, los otros (es decir los pares), y ellos (constituido por los docentes), generando como resultado el control y el gobierno de los cuerpos de los cuales se producen subjetividades moduladas. Esto permite proporcionar ciertos conocimientos que posibilitan determinadas acciones encaminadas a disminuir las problemáticas que las/los jóvenes y adolescentes generan a la sociedad, en este caso, producto de sus prácticas 
sexuales y/o reproductivas: fecundidad, morbilidad, embarazos prematuros y mortalidad, cuyos efectos ya no son tanto disciplinantes y normalizadores sino de autoregulación, generando el espejismo de la autonomía para ejercer su vida sexual pero de manera "segura", "sana" y "responsable". Esto implica retardar la edad de inicio de su vida sexual, procurar parejas estables y evitar los embarazos adolescentes.

Estas diversas formas de vigilancia son desplegadas en torno a los comportamientos y conductas sexuales de las/los jóvenes y adolescentes y se sustentan en "la transferencia que se les hace de las decisiones relativas a la administración y protección de su salud, el control de su reproducción, la construcción de su proyecto de vida, la escogencia de su estilo de vida, etc." ${ }^{12}$ (Viveros, 2006, 151).

Ahora bien, en educación para sexualidad y construcción de ciudadanía hay un cambio de centro. Aunque los mecanismos parten desde afuera, pues es desde allí donde se ofrecen todos los lineamientos y condiciones para que el sujeto realice un trabajo interno que luego lo vivencia por sí misma/o, es ahora ella/él quien se autogobierna, quien se auto restringe. Es a través de los procesos de reflexión y autonomía que ella/él llega al mismo punto que se quería llegar desde afuera: llevar una sexualidad sana, responsable y placentera. Lograr una disminución de los riegos, retrasar las edades de inicio sexual, decidir el número de hijos, reconocer los beneficios de la pareja estable.

En términos de ciudadanía, se propende ya no por una internalización autómata de la norma sino por la comprensión y el ejercicio con pleno conocimiento. En competencias ciudadanas, por ejemplo, se espera propiciar un ambiente democrático en la escuela en el que se valore y comprenda la norma social y personal para que de esta manera se obtenga sanción social por incumplimiento pero con posibilidad de reparación y restauración (MEN, 2004). Bien ¿cuáles normas?, la respuesta es evidente: las preestablecidas. En este sentido la instrumentalización del consenso genera el espejismo de participación en/de los órdenes fijados por otros.

Las nociones como autonomía -sexual y social- y responsabilidad -sana en el ejercicio sexual- que aparecen en las propuestas de educación sexual y construcción de ciudadanía, van encaminados a lograr la autovigilancia de los cuerpos y de los comportamientos genéricos y sociales en la escuela. Prácti- 
cas que afianzan los imaginarios y representaciones de la cultura dominante que moldean las subjetividades puesto que las lógicas del género y, también, de ciudadanía en este caso no consisten en estructuras externas a los sujetos, se les imponen en un sistema de regulación patriarcal y exclusivo. Los objetivos son loables, no hay que negarlo, pero van encaminados a preservar el orden social existente. Así, los discursos de la sexualidad oscilan entre lo que Viveros llama dispositivos de seguridad y disciplinamiento, es decir se transita entre una intervención externa y unos controles internos producto de los efectos de los discursos preexistentes (Viveros, 2006).

Para los grupos emergentes, al igual que para las mujeres, el cuerpo es el primer territorio de lucha (Foucault, 2005; Spivak, 1987):

cuerpo como territorio donde se materializa la subjetividad, materialización en la cual los cuerpos operan a la vez como objetos de control y disciplina, y como agentes mediante los cuales realizamos lo que somos o queremos ser. En torno a ellos, se activa un conjunto de prácticas culturales que se orientan a la producción de imaginarios diferenciados sobre el ser y el deber ser de las personas, y a los modos de normalización y control de sus fronteras (García, 2007, 24).

Salirse de los estereotipos y modelos autorizados para lograr construir un cuerpo nuevo y "propio" es aún la lucha en la que permanecen estos grupos, pero los campos lingüísticos y semióticos están contaminados por las representaciones hegemónicas, que se refuerzan en estos programas y que la escuela se encarga de mantener.

No obstante, el poder transformador que también porta la escuela es una vía para la construcción y reconocimiento de nuevas figuraciones (Braidotti, 2000; 2004). El reto está en desmantelar las matrices que constriñen los marcos de acción, posible a través de la incorporación de debates y producciones teóricas a las prácticas y relaciones educativas. Habría que partir también, como indicaba Foucault, de los mecanismos infinitesimales para desmontar las lógicas de poder y dar paso a las nuevas relaciones de poder creativas e innovadoras en las que las representaciones aquilatadas y fosilizadas sean movilizadas por nuevas figuraciones, sujetos emergentes y estilos de vida diferentes. Es decir, la escuela puede "coordinar y dar una finalidad" a las potencias que transitan diariamente por sus recintos, ya no 
como poder-saber soberano que determinaba y era determinante en la constitución de subjetividades estables.

\section{Discursos sobre ciudadanía}

Por su parte, los discursos acerca de la ciudadanía se dan en términos de derechos, valores y principios. En la primera línea se subrayan los Derechos Humanos, derechos de los niños -según sea el grupo etáreo al que se dirige- y los Derechos Sexuales y Reproductivos. De esta manera, como se ha reconocido en repetidas ocasiones, se mantiene un discurso legal que ha reconocido un sujeto universal y asexuado: el hombre. Es el mismo discurso que dio origen a parte de las demandas reivindicativas de las mujeres ante el malestar generado por la noción de igualdad que no incorpora ningún tipo de diferencia por considerarlas poco relevantes. Hasta el día de hoy las luchas de los grupos sexuales emergentes han tenido que librarse legalmente en los términos de igualdad relegando el tema de la diferencia a las luchas culturales (Butler, 2000).

El origen conceptual de los Derechos Reproductivos corresponde a una elaboración teórica necesaria para fundamentar y tratar de construir nuevas estructuras sociales favorables a la maternidad y a la paternidad libre y responsable ${ }^{13}$. Vemos que sea cual sea el esfuerzo por restituir derechos y garantías, se da en términos homogeneizantes y no pretenden revertir ni cuestionar realmente el orden establecido.

En cuanto a los valores y principios, se transita entre estos dos términos, el primero para reforzar el carácter moral y ético, y el segundo como aspecto que acompaña la propuesta de los derechos. Dentro de este grupo quiero resaltar el valor / principio de la tolerancia, debido a que está presente tanto en el discurso de valores como en el de principio y se promueve en todos los programas. Como valor en su acepción negativa, en ocasiones se confunde con la "promoción de", noción que se desprende de los alegatos religiosos y moralizantes para obstaculizar la enseñanza de la educación para el ejercicio de las sexualidades comprensivas en la que se cree estar incitando a las/los adolescentes y las/ los jóvenes a la práctica indiscriminada de las relaciones genitales y la incitación a comportamientos homosexuales. Este sentir se percibe en la presentación realizada por Erik Cantor al trabajo de la Corporación Promover Ciudadanía y en las/los docentes 
en las mesas de trabajo de los colegios visitados. Para controlar la situación se recomiendan estrategias para seguir influyendo y gobernando los cuerpos, "inevitablemente, en cierta medida el discurso moralista sobre 'promiscuidad' se recicló adquiriendo ropajes técnicos, y se comenzó a promover la 'reducción del número de parejas sexuales', fuera de, ciertamente, la práctica de 'sexo más seguro', cuando no eran posibles la abstinencia o la monogamia" (Cáseres, 2004, 39).

El concepto de tolerancia también porta connotaciones un tanto ambiguas que pueden terminar siendo desfavorables, como aceptar/soportar/aguantar a alguien/algo: "los grandes esfuerzos [de Profamilia] van dirigidos a generar tolerancia y aceptación a las diferentes opciones sexuales, que se ven en el interior de la institución, tanto en el discurso institucional como en las prácticas de sus funcionarios en los programas que se llevan a cabo en Cali" (Profamilia, 2005). De esta manera existe el riesgo de que las propuestas no superen el llamado a la tolerancia y se reduzca todo a la estrategia de condescendencia (Bourdieu, 2000), pues la simple incitación a la tolerancia no representa cambios profundos, ni respeto y reconocimiento de las diferencias y aunque se haga mención, éstas van aparejadas de "valores" que han obstaculizado la acción de las mujeres, valores como lo son el amor, romántico principalmente (Giddens, 1998):

En términos formales, el PNES ha buscado: propiciar la autonomía, la autoestima, la convivencia y los estilos de vida saludables, de una manera dinámica, dialogal y permanente, legitimando un espacio en el ámbito escolar que permita la reflexión frente a la sexualidad para construir de manera colectiva formas de relación dentro de una cultura tolerante, creativa y respetuosa por la diferencia que haga posible el desarrollo del amor y de la vida (Cantor, 2008, 126).

La relación entre las sexualidades y los afectos también es problemática. Por lo menos en la cultura occidental, la relación amor-sexualidad ha estado marcada y cargada por una historia jerarquizada que ha conllevado desventajas para las mujeres. Este vínculo define características diferentes para las sexualidades femeninas y masculinas, en las que aparece un acento marcado del amor-pasión como característica masculina y amorromance como cualidad femenina (Loyola, 1998, Cit. en Szasz; Giddens, 1998). Presentar esta relación como universal es desconocer la marca histórica y las divergencias culturales (Szasz, 
2004), asimismo subrayarla demasiado puede acentuar esta jerarquización pues no se han construido nuevas figuraciones; las variaciones consideradas, en realidad, han sido concesiones dadas a los varones de mantener varias relaciones eróticas sin compromisos emocionales, mientras a las mujeres se les mira mal cuando construyen prácticas semejantes.

Entonces, junto a la lógica de derechos humanos subyacen las bases para garantizar libertad, equidad, diversidad y respeto: la autodeterminación y la responsabilidad con sí mismo, los demás y el entorno. Estos mecanismos hacen parte de una pedagogía en la que hay una enfermiza preocupación por hacer de los cuerpos infantiles y juveniles cuerpos "bien educados", que "guarden la compostura", que no sean "inquietos", que se moldeen para que puedan aguantar en silencio y con trabajo largas jornadas de clases, mientras aprenden a sentir y a moverse con los parámetros establecidos. El sistema educativo ha formado "estilos de cuerpos disciplinados e higienizados", aptos para rendir en el mundo del mercando, de la estética dominante y del orden social existente; es decir, cuerpos social y políticamente "correctos" (Foucault, 2005; González-Stephan, 1998; Cantor, 2008).

Dentro de la investigación también se establecieron aspectos que constriñen/limitan los mismos discursos sobre las sexualidades y las ciudadanías, tales como el concepto de Familia -legitimado constitucionalmente como aquella conformada voluntariamente por un hombre y una mujer con el objetivo claro de reproducir la prole (CP. 1992, Art. 42)- y proyectos de vida -los cuales son formulados desde una posición "adultocentrista", siendo así los adultos (profesionales de diversas áreas, profesoras/es y el aparato estatal) los encargados de designar, desde sus perspectivas, las posibilidades de acción de los y las jóvenes para lograr una sexualidad sana, responsable y placentera-. De igual forma, se determinaron discursos de cambio conceptual en las que una permuta preposicional o sutantivacional marca el viro: “De la educación 'en' sexualidad a la educación 'para' la sexualidad y De la sexualidad como aspecto/cualidad a la sexualidad como condición/dimensión. Finalmente, al interior de los discursos establecidos hay algunos que soportan lógicas binarias, puesto que detrás del placer está el riesgo, detrás de la responsabilidad sexual está la ausencia o disminución de prácticas sexuales, detrás de los derechos están los deberes.

En suma, vemos cómo en los planes y programas para la 
educación sexual y construcción de ciudadanía estatales, fundamentalmente, se despliegan discursos y pedagogías para continuar controlando los cuerpos y los comportamientos. Ahora bajo la lógica de la auto-soberanía y/o autogobierno se desplaza el centro de poder de un lugar exógeno a uno endógeno para alimentar los espejismos de libertad y autonomía. Sin embargo, continúan siendo máscaras del poder como dominio de las posibilidades de acción de las y los otras/os. Mientras las propuestas emergentes, sin ser decididamente radicales, aún permanecen entre una relación de resistencia y creación pues todavía median con una cuestión de supervivencia política (Lazzarato, 2007).

Antes de cerrar este texto, es atinente referir algunas de las conclusiones a las cuales llegó la investigación. En primera instancia, en Colombia el campo de las sexualidades está dominado principalmente por los discursos biológicos, médicos y psicológicos, los cuales están encaminados a lograr un autogobierno de los cuerpos (control) y de las subjetividades más que una comprensión de los mismos.

En términos generales, se constata que no ha habido transformaciones reales del orden sexual en el sector educativo colombiano. Aunque las presiones generadas por las/los sujetos emergentes, así como las luchas y demandas que mantienen han flexibilizado las fronteras/los límites restrictivas/os de las sexualidades, éstas siguen confiscadas en las lógicas de la normalización, los comportamientos adecuados y los valores apropiados. Se ha reconocido un carácter construido pero no ha sido fácil deshacer discursos, representaciones y prácticas pues "en las últimas tres o cuatro décadas del siglo XX asistimos a cambios en la sexualidad que corresponden más a una dinámica de individualización de los comportamientos y los ideales que a una revolución sexual, en el sentido de una transformación radical del orden Sexual" (Viveros, 2006, 152). Hay esfuerzos e intereses pero no han sido suficientes, pierden la fuerza y el entusiasmo con los cambios de gobierno y con el agotamiento de los financiamientos.

Los enfoques de educación ciudadana, por su parte, deben superar el tono normativizador y normalizador, y canalizar los propósitos para aumentar la potencia de actuar, intersecando lo social, lo político y lo ético, para que cada sujeta/o pueda determinar las condiciones, las posibilidades y las justificaciones de las acciones (Fazio, 2007). También deben superen el llamado a 
la tolerancia puesto que ésta no garantiza procesos de reconocimiento sustantivo y la comprensión profunda de las diferencias. De igual forma, es necesario que estas iniciativas favorezcan la participación activa de las/los adolescentes y jóvenes, pues ello permitiría configurar procesos de afirmación y defensa de sus derechos -no solo sexuales y reproductivos-, lo que contribuiría a superar las contradicciones entre un discurso que busca promover las capacidades reflexivas, autónomas y las actitudes responsables y activas dirigidas al autocuidado.

Un programa de educación para las sexualidades y las ciudadanías además de tener en cuenta las particularidades contextuales, en el caso de Colombia o de cualquier otro país de América Latina, debe abogar por la construcción histórica y cultural de las mismas.

\section{Notas}

1 Este texto hace parte de la investigación realizada para optar al título de Magíster en Estudios de Género y Cultura, mención Humanidades de la Universidad de Chile, la cual se basa en un "Análisis de las propuestas de educación sexual y construcción de ciudadanía existentes en Colombia desde una perspectiva crítica de género". Esta tesis cuenta con el apoyo de la profesora Margarita Iglesias Saldaña.

2 Para el caso a revisar los Ministerios de Educación Nacional (MEN) y el Ministerio de Protección Social (MINPROTECCIÓN) en Colombia.

3 El objetivo principal de la investigación es develar los discursos latentes/subyacentes en las nociones de sexualidades y ciudadanías a la luz de las propuestas teórico-conceptuales sobre perspectiva de género, cuerpo, sexo y sexualidad, por un lado; y ciudadanía, conocimiento y ejercicio de derechos, inclusión y diferencia, por otro, para realizar los respectivos análisis e interpretaciones, y finalmente generar sugerencias de formación docente inicial alternativa

4 La Constitución Política de 1991 y la Ley General de Educación, Ley 115 de 1994- para determinar los enfoques y el nivel de importancia que se le otorga a los temas de Educación Sexual y Ciudadana desde las Políticas de Estado. En esta misma línea se encuentran los programas del Ministerio de Educación Nacional (MEN) emanados de estas disposiciones desde 1992 a la fecha: Plan Nacional de Educación Sexual (PNES 1993; 1999), Competencias Ciudadanas (2004) y Programa Nacional de Educación 
Sexual y Construcción de Ciudadanía (PESCC) 2006-2008.

5 Para el caso se revisaron dos propuestas: una realizada por Colombia Diversa (2007) y la otra realizada por Promover Ciudadanía (2008), organizaciones no gubernamentales que trabajan en defensa de los derechos de la población Lesbiana, Gay, Bisexual y Transexual (LGBT)

6 Al respecto ver Dáguer, Carlos y Marcelo Riccardi. Al derecho y al revés. La revolución de los derechos sexuales y reproductivos en Colombia. Bogotá: Profamilia, 2005 y Cardinal de Martín, Cecilia. Educación Sexual. Un proyecto humano de múltiples facetas. Bogotá: Siglo del Hombre Editores, 2005.

7 En Colombia se empiezan a dar los debates para incluir la I de Intersexual, en el grupo de la población LGBT. En Brasil ya se ha ampliado el grupo y se ha incorporado al s (LGBTs) de simpatizante.

$8 \quad$ El énfasis es del original.

9 Esta propuesta cambia la estrategia de ejes por hilos conductores los cuales comprenden componentes y funciones de la sexualidad, hilos y competencias que deben ser desarrolladas por las y los estudiantes "Cada componente y función de la sexualidad tiene una serie de hilos conductores, que reunidos comprenden los principales aspectos que debe abordar el sistema de educación preescolar, básica y media con respecto a la sexualidad. A cada hilo le corresponde, a su vez, una competencia general, que condensa las habilidades, las actitudes y los conocimientos que todas las personas deben haber alcanzado al completar su educación media" (MEN-UNFPA, 2008, módulo 2, 8).

10 Ejemplo de competencia que debe desarrollar la y el estudiante en el componente de función reproductiva.

11 Profamilia, Asociación Pro-Bienestar de la Familia Colombiana, es una entidad privada sin ánimo de lucro, con más de cuarenta años de servicio en el país. Esta institución que, aunque no es Estatal, representa la voz autorizada en Salud Sexual y Reproductiva. Cuenta con el auspicio y el financiamiento de la Federación Internacional de Planificación Familiar (IPPF, sigla en inglés) y brinda el $65 \%$ de los servicios de Planificación en Colombia a través de cuarenta centros de atención a nivel nacional. Profamilia Joven inició en el año 1990 y es un Programa de Atención Integral Especializado en Sexualidad y Derechos Sexuales y Reproductivos para todas las personas menores de 20 años. El trabajo de base es ofrecer información a las y los jóvenes que voluntariamente se acercan a sus oficinas, también realizan talleres informativos a las organizaciones e instituciones escolares que los contactan, pero su actividad principal es prestar servicios a bajo costo. Al respecto ver Dáguer y Riccardi, 2005.

12 Ver Política de Salud Sexual y Reproductiva 2002-2006, y los pro- 
gramas de Profamilia Joven.

13 Ver Política Nacional de Salud Sexual, Ministerio de Protección Social, 2002-2006.

\section{Bibliografía}

Bourdieu, Pierre. La dominación masculina. Barcelona: Anagrama, 2000. Braidotti, Rosi. Sujetos nómades. Buenos Aires: Paidós, 2000. . Feminismo, diferencia sexual y subjetividad nómade. Barcelona: Gedisa, 2004.

Butler, Judith. "Variaciones sobre sexo y género: De Beauvoir, Wittig y Foucault". Teoría Feminista y teoría crítica. Seyla Benhabid, y Drucilla Cornella, compiladoras. Valencia: Ediciones Alfonso el Magnánimo, 1990. 193-211.

. "Actos performativos y constitución del género: un ensayo sobre fenomenología y teoría feminista". Debate Feminista. 9, Vol., 18, (1998): 296-314.

. "El Marxismo y lo meramente cultural". New Left Review. 2, (2000): 109-120.

Cantor, Erik La homofobia en la escuela. Bogotá: Promover Ciudadanía, 2008.

Cullen, Carlos A. (comp.) El malestar de la ciudadanía. Buenos Aires: La Crujía, 2007.

Cardinal de Martín, Cecilia. Educación Sexual. Un proyecto humano de múltiples facetas. Bogotá: Siglo del Hombre Editores, 2005.

Cáseres, Carlos et al. Eds. Ciudadanía Sexual en América Latina: Abriendo el Debate. Perú: Universidad Peruana Cayetano Heredia, 2004.

Colombani, María Cecilia. Foucault y lo político. Buenos Aires: Prometo, 2009.

Dáguer, Carlos y Marcelo Riccardi. Eds. Al derecho y al rev[é]s. Bogotá: Profamilia, 2005.

Foucault, Michel. Historia de la sexualidad. La voluntad del saber. Vol. I. España: Siglo Veintiuno Editores, 2005.

. Historia de la sexualidad. El uso de los placeres. Vol. II. Siglo Veintiuno Editores, España, 1987.

Microfísica del poder. Ediciones de La Piqueta, Madrid, 1992.

El orden del discurso. Tusquets Editores, Barcelona, 2002.

García Suárez, Carlos Iván. Ed. Hacerse hombres, hacerse mujeres. Dispositivos pedagógicos de género. Santafé de Bogotá: Siglo del Hombre Editores, 1998.

- Proyecto Arco Iris: Una mirada transformadora a las relaciones de género en la escuela. Departamento de Investigación de la Universidad Central, Bogotá, 2003.

Diversidad Sexual en la Escuela. Dinámicas pedagógicas para enfrentar la homofobia. Bogotá: Colombia Diversa, 2007.

Giddens, Anthony. La transformación de la intimidad. Sexualidad, amor y 
erotismo en las sociedades modernas. Madrid: Cátedra, 1998.

González-Stepahan, Beatriz. "Cartografía de la sociedad disciplinaria. Antesala de la sociedad de control en Venezuela". Revista de la Universidad Javeriana 1998 http://www.javeriana.edu.co/pensar/ Rev42.html

Lamas, Marta. “Usos, dificultades y posibilidades de la categoría género" El género: La construcción cultural de la diferencia sexual. Marta Lamas, compiladora. México: UNAM, 1996. 326-366.

Lazzarato, Maurizio. "Del biopoder a la biopolítica". Caosmosis 2007 http: / / caosmosis.acracia.net/ $\mathrm{p}=423$

Ministerio de Educación Nacional. Plan Nacional de Educación Sexual. Bogotá: MEN, 1993, 1994, 1999.

. Competencias Ciudadanas. Guía No 6. MEN, Bogotá, 2004.

. Proyecto Piloto para la Educación en Sexualidad y construcción de Ciudadanía. Hacia una Política Pública. Bogotá: MEN-UNFPA, 2006.

. Programa Nacional de Educación para la Educación en Sexualidad y construcción de Ciudadanía. Bogotá: MEN-UNFPA, 2008.

Ministerio de Protección Social. Política de Salud Sexual y Reproductiva 2002-2006. Bogotá: Minprotección, 2003.

Programa Presidencial para la Juventud, la Mujer y la Familia. Plan Nacional de Educación Sexual de la República de Colombia. Bogotá: PPJMF, 1992.

Promover Ciudadanía. Lineamientos Pedagógicos para la sexualidad y los derechos sexuales y reproductivos. Bogotá: Promover Ciudadanía, 2008.

Rich, Adrienne. "La heterosexualidad obligatoria y la existencia lesbiana". Sexualidad, género y roles sexuales. Marysa Navarro y Catharine Stimpson, editoras. Buenos Aires: Fondo de Cultura Económica, 1999. 159-211.

Szasz, Ivonne. "El discurso de las Ciencias Sociales sobre las sexualidades". Ciudadanía Sexual en América Latina: Abriendo el Debate. Carlos Cáseres et al, editores. Perú: Universidad Peruana Cayetano Heredia, 2004.

Viveros, Mara. "Políticas de sexualidad juvenil y diferencias étnicoraciales en Colombia: reflexiones a partir de un estudio de caso". Estudios Feministas, Florianópolis, 2006.

VV.AA. Ley General de Educación. Ley 115 de 1994. Bogotá: Unión, 1994.

VV.AA. Constitución Política de Colombia. Bogotá: El Pensador Ltda., 1999. 